LBL-35821

\title{
Consequences of Nuclear Shadowing for Heavy Quarkonium Production in Hadron-Nucleus Interactions ${ }^{\star}$
}

\author{
S. Liuti \\ Nuclear Science Division, Lawrence Berkeley Laboratory, Berkeley CA 94720, USA \\ and \\ I.N.F.N. Sezione Sanitá \\ Viale Regina Elena 299, I-00161 Rome, Italy \\ R. Vogt \\ Nuclear Science Division, Lawrence Berkeley Laboratory, Berkeley CA 94720, USA
}

\begin{abstract}
We study nuclear shadowing in $J / \psi$ and $\Upsilon$ production in hadron-nucleus interactions and in nucleus-nucleus collisions at the Relativistic Heavy Ion Collider and the Large Hadron Collider. As a consequence of the perturbative $Q^{2}$-dependence of gluon shadowing, we predict that $\Upsilon$ production is less suppressed than the $J / \psi$. We show that antishadowing leads to enhanced $J / \psi$ production at $x_{f} \lesssim 0$, an effect reduced for $\Upsilon$ production.
\end{abstract}

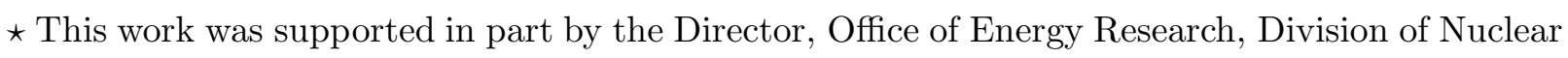
Physics of the Office of High Energy and Nuclear Physics of the U. S. Department of Energy under Contract Number DE-AC03-76SF0098.
} 
The production of charmonium and bottomonium states in hadron-nucleus interactions does not increase linearly with the nuclear target size $A$. Additionally, the ratio of hadronnucleus to hadron-nucleon production, $S_{A}=\sigma_{h A} / A \sigma_{h p}$, is not constant but decreases as a function of the fraction of the center of mass momentum, $x_{f}$, carried by the produced resonance. There are several effects that contribute both to the $A$ dependence of the total cross section and to the ratio $S_{A}$ including nuclear absorption [1], comover interactions [2], intrinsic heavy quark states [3], projectile energy loss [4], and nuclear shadowing [5; 6].

In this paper, we examine the role of shadowing of the nuclear parton distributions in quarkonium production. Nuclear shadowing modifies the target parton distributions so that $x q^{A}(x) \neq A x q^{N}(x)$. Deep inelastic scattering (DIS) data on the ratio $R_{A}=F_{2}^{A} / F_{2}^{D}$ [7, [8] show that: $i)$ nuclear shadowing begins to set in at $x_{B j} \lesssim 0.06$ for all nuclei $\left(R_{A}<1\right)$; ii) $R_{A}$ has a very weak $Q^{2}$ dependence; and iii) an enhancement, or antishadowing, exists for $0.06 \lesssim x_{B j} \lesssim 0.25\left(R_{A}>1\right)$. In addition, the NMC data on $J / \psi$ production with Sn and C targets show an enhancement of $(13 \pm 8) \%$ for $0.05 \lesssim x_{B j} \lesssim 0.3$ [9], interpreted as gluon antishadowing.

The weak $Q^{2}$ dependence of $R_{A}$ is consistent with the models of [10,[1], where nuclear shadowing is considered as a leading-order QCD effect with perturbatively generated $Q^{2}$ dependence. These models differ in the treatment of the nonperturbative contribution at the initial scale $Q_{0}^{2}$. We adopt the QCD Aligned-Jet Model (QAJM) [11] which accounts for the DIS data [11,12] to study the effect of nuclear shadowing on $S_{A}$.

We would like to point out two major consequences of shadowing and antishadowing for $J / \psi$ and $\Upsilon$ hadroproduction based on very general arguments. Since $m_{\Upsilon}$ is three times larger than $m_{\psi}$, the $\Upsilon$ is primarily produced within the antishadowing region of the target at present energies. However, the parton distributions are evaluated at $Q^{2}=m_{\Upsilon}^{2}$, thus $Q^{2}$ evolution reduces the effect of both shadowing and antishadowing in $\Upsilon$ production with respect to $J / \psi$. The antishadowing apparently present in both the DIS measurements of $R_{A}$ [7.8] and the gluon distribution [9] reflects on $S_{A}$. We include the observed antishadowing effect, a general consequence of baryon number and momentum conservation in a nucleus, 
and explore the consequences of $Q^{2}$ evolution on $S_{A}$.

We do not expect our shadowing model to fully explain the shape and magnitude of $S_{A}$. To quantitatively show the effects of nuclear shadowing, we have not included other $A$ dependent effects. Nuclear absorption and comover interactions do not have a strong $x_{f}$ dependence and mainly affect the magnitude of $S_{A}[\mathbb{1}, 2]$. On the other hand, intrinsic heavy quarks [3] and projectile energy loss [4] have a significant $x_{f}$ dependence.

The leading order $Q \bar{Q}$ bound state cross section is the integral of the free $Q \bar{Q}$ production cross section from the $Q \bar{Q}$ threshold to the meson pair threshold,

$$
\frac{d \sigma}{d x_{f}}=2 F \int_{2 m_{Q} / \sqrt{s}}^{2 m_{H} / \sqrt{s}} \tau d \tau \frac{H_{p t}\left(x_{1}, x_{2} ; x_{1} x_{2} s\right)}{\sqrt{x_{f}^{2}+4 \tau^{2}}},
$$

where $m_{c}=1.5 \mathrm{GeV}, m_{b}=4.75 \mathrm{GeV}, m_{D}=1.867 \mathrm{GeV}$, and $m_{B}=5.28 \mathrm{GeV}$. The bound state fraction, $F$, is a parameter that cancels in $S_{A}$. We include quark-antiquark annihilation and gluon-gluon fusion subprocesses in the convolution formula $H_{p t}$,

$$
\begin{aligned}
H_{p t}\left(x_{1}, x_{2} ; m^{2}\right)= & G_{p}\left(x_{1}\right) G_{t}\left(x_{2}\right) \sigma\left(g g \rightarrow Q \bar{Q} ; m^{2}\right) \\
& +\sum_{q=u, d, s}\left(q_{p}\left(x_{1}\right) \bar{q}_{t}\left(x_{2}\right)+\bar{q}_{p}\left(x_{1}\right) q_{t}\left(x_{2}\right)\right) \sigma\left(q \bar{q} \rightarrow Q \bar{Q} ; m^{2}\right),
\end{aligned}
$$

where $x_{1,2}=\frac{1}{2}\left( \pm x_{f}+\sqrt{x_{f}^{2}+4 \tau^{2}}\right)$ are the projectile and target momentum fractions. We use the MRS D-' [13] distributions that describe the recent HERA data on $F_{2}^{e p}$ [14].

Next-to-leading order contributions to the free $Q \bar{Q}$ cross section have been calculated. The $x_{f}$ distribution is approximately that from lowest order multiplied by a theoretical $K$ factor, $\sigma\left(\alpha_{s}^{3}\right) / \sigma\left(\alpha_{s}^{2}\right)$ [15], assumed to be absorbed in $F$. At lowest order, the $J / \psi$ is produced with $p_{T}=0$ since the $c \bar{c}$ pair is back-to-back. A $p_{T}$ dependence may be introduced through an intrinsic transverse momenta, $q_{T}$, of the initial partons as we show later. For $p_{T} \gg m_{\text {res }}$, higher-order corrections are needed [16]. Since we concentrate on the $x_{f}$ distributions and the $A$ dependence, where $p_{T} \lesssim m_{\text {res }}$ is dominant, our calculation is lowest order.

When the target is a nucleus, the parton distributions, $G_{t}\left(x_{2}\right), q_{t}\left(x_{2}\right)$ and $\bar{q}_{t}\left(x_{2}\right)$ in Eq. (2) are the nuclear medium modified distributions evaluated at $Q^{2} \approx m_{\psi}^{2}$ and $Q^{2} \approx m_{\Upsilon}^{2}$ for $J / \psi$ and $\Upsilon$ production. We calculate the shadowed distributions at the initial scale, $Q^{2}=$ 
$Q_{0}^{2} \approx 5 \mathrm{GeV}^{2}$, and $x<x_{\text {sh }} \approx 0.04$ according to the QAJM [11] and evolve in $Q^{2}$ using the Dokshitser-Gribov-Lipatov-Altarelli-Parisi equations (DGLAP) [17]. Recombination effects within a single nucleon are negligible at $x \gtrsim 10^{-3}$ [5,18]. In the QAJM, shadowing is generated by the interaction of hadronic configurations originating from fluctuations of the virtual photon with small transverse momentum, or jets aligned along the direction of the virtual photon, $\overrightarrow{\mathbf{q}} /|\mathbf{q}|$, with $k_{\perp}<k_{0 \perp} \approx 0.4 \mathrm{GeV}$. The transverse separation of the aligned jets is correspondingly large $(\approx 1 \mathrm{fm})$ and their phase space is restricted by a factor $\propto$ $k_{0 \perp}^{2} / M^{2}$. In QCD the transverse momentum of the fluctuation can be large so that nonaligned hadronic configurations are simultaneously present. Such configurations interact differently in the nuclear medium. In Refs. [11, 12] it was assumed that aligned jets with large transverse separation interact in the nucleus as vector mesons. Nonaligned jets, with $k_{\perp}>k_{0 \perp}$ and a small transverse separation have a correspondingly small interaction cross section, giving rise to color transparency.

The nuclear baryon number and momentum sum rules:

$$
\begin{aligned}
& \frac{1}{A} \int_{0}^{A} d x V_{A}\left(x, Q^{2}\right)=\int_{0}^{1} d x V_{N}\left(x, Q^{2}\right), \\
& \frac{1}{A} \int_{0}^{A} d x x\left[V_{A}\left(x, Q^{2}\right)+G_{A}\left(x, Q^{2}\right)+S_{A}\left(x, Q^{2}\right)\right]= \\
& \int_{0}^{1} d x x\left[V_{N}\left(x, Q^{2}\right)+G_{N}\left(x, Q^{2}\right)+S_{N}\left(x, Q^{2}\right)\right],
\end{aligned}
$$

must be fulfilled at every $Q^{2}$. In Eq. (3b) $V_{N(A)} \equiv u_{t}^{V}+d_{t}^{V}, S_{N(A)} \equiv 2\left(\bar{u}_{t}+\bar{d}_{t}+\bar{s}_{t}\right)$, and $G_{N(A)} \equiv G_{t}$, are the nucleon, $N$, and nuclear, $A$, valence, sea and gluon distributions. In order to satisfy the sum rules and simultaneously allow for shadowing at $x \lesssim 0.04$, the parton distributions must be enhanced at higher values of $x$. We assume that the enhancement is shared by the valence quarks and gluons since the sea quark enhancement is consistent with zero [19] (see also Ref. [12]). Recent data from E665 [8] show that the enhancement in $R_{A}$ is concentrated in the interval $0.06 \lesssim x \lesssim 0.25$ and is $A$-independent. These two crossover points, assuming no sea enhancement, are sufficient to constrain the valence and gluon antishadowing.

Gluon shadowing should be larger than sea quark shadowing since the interaction of 
a $q \bar{q} g$ configuration in the $\gamma^{*}$ wavefunction with the target should be stronger than a $q \bar{q}$ configuration [20,21]. Indeed, the perturbative interaction cross section of a small color octet with a nucleon is 9/4 larger than a color triplet of the same transverse size. Assuming a smooth connection between the perturbative and nonperturbative domains, $\sigma_{\gamma^{*} \rightarrow q \bar{q} g, N}=$ $\frac{9}{4} \sigma_{\gamma^{*} \rightarrow q \bar{q}, N}$, leading to larger effects on the gluon relative to the sea and valence quarks [20]. The overall enhancement found in [12,20] is consistent with the data of [9], used to constrain the shape of the gluon distribution in the antishadowing region. A weak $Q^{2}$ dependence for $R_{A}$ is obtained, in accord with the data of [7, [8], while a stronger $Q^{2}$ dependence is found for the gluon ratio, $R_{A}^{G}=G_{A} / G_{N}$. Similar results were also obtained in [22].

In Fig. 1 we show $x_{2}$ as a function of $x_{f}$ for $J / \psi$ (solid curve), $\Upsilon$ (dashed) production for several energies to point out the shadowing and antishadowing regions. At $200 \mathrm{GeV}, 1$ (a), the $J / \psi$ is antishadowed for $0 \lesssim x_{f} \lesssim 0.6$. At $800 \mathrm{GeV}, 1(\mathrm{~b})$, where data exists for $J / \psi$ and $\Upsilon$ from $-0.2<x_{f}<0.65$ [23], the $J / \psi$ lies in the shadowing region for $x_{f}>0$ but enters the antishadowing region at backward values. The $\Upsilon$ is mainly produced in the antishadowing region, but when $x_{f}<0$, it is in the EMC region. Since the parton distributions are evaluated at $m_{\Upsilon}^{2}$ and the gluon distribution evolves faster than the quarks, we expect $S_{A}^{\Upsilon}>S_{A}^{\psi}$ for $x_{f}>0$. In $1(\mathrm{c})$ and $1(\mathrm{~d})$, the $x_{f}$ range corresponds to $-0.35<y<2.5$ and $|y|<1$, the rapidity coverage of the PHENIX [24] and ALICE [25] detectors at RHIC $(\sqrt{s}=200 A \mathrm{GeV})$ and the LHC $(\sqrt{s}=5.5 \mathrm{TeV})$, respectively. At RHIC central rapidities the $\Upsilon$ may still be antishadowed, but would be detected in the shadowing region with the muon spectrometer [24]. At the LHC, both $J / \psi$ and $\Upsilon$ production are shadowed.

The shadowing and antishadowing features of $S_{A}$ are shown explicitly in Fig. 2 where we compare $S_{A}^{\psi}$ and $S_{A}^{\Upsilon}$ to the $\mathrm{E} 772$ data at $800 \mathrm{GeV}$ on C, $\mathrm{Ca}, \mathrm{Fe}$, and $\mathrm{W}$ targets [23]. Recall that when $x_{f}$ is increasing, $x_{2}$ is decreasing. The solid curves show $S_{A}^{\psi}$, the dashed, $S_{A}^{\psi^{\prime}}$, and the short dashed, $S_{A}^{\Upsilon}$. The difference between $J / \psi$ and $\psi^{\prime}$ comes from their small mass difference. Antishadowing is clearly seen for the $J / \psi$ as $x_{f} \rightarrow 0$. Antishadowing at negative $x_{f}$ is in contradiction with the data: clearly other effects are needed to account for this behavior. The effect of $Q^{2}$ evolution on $S_{A}^{\Upsilon}$ is apparent-the antishadowing peak is 
broader and closer to unity than the corresponding region of $S_{A}^{\psi}$. The $\Upsilon$ enters the EMC region for $x_{f}<0$, decreasing $S_{A}^{\Upsilon}$. While this trend agrees with the shape of the data, the magnitude is too large.

Accounting for the primordial transverse momentum of the partons, $q_{T}$, may reduce the magnitude of $S_{A}$ since the transverse momentum spectrum may be broadened in a nucleus because of Fermi motion. If the cross sections are integrated over all $p_{T}$, the $q_{T}$ smearing will have no effect (the $q_{T}$ spectrum in a moving nucleon has the same normalization as for a stationary one). However, the data is taken over a finite range of $p_{T}$, suggesting that the broader $q_{T}$ smearing in the nucleus manifests itself in a reduced $p_{T}$-integrated cross section relative to the nucleon, reducing $S_{A}$. We calculated the $q_{T}$ spectra in nuclei by smearing the primordial transverse momentum distribution in a free nucleon with nucleon momentum distributions from realistic many-body calculations [26]. The primordial $q_{T}$ spectrum for a free nucleon was parametrized by a gaussian with $\left\langle q_{T}^{2}\right\rangle \approx 1 \mathrm{GeV}^{2}$, the same form used to describe the low $p_{T}$ Drell-Yan data in $p p$ scattering [28]. Similar values of $\left\langle q_{T}^{2}\right\rangle$ are also compatible with the low $p_{T}$ data for $J / \psi$ production 29]. The convolution formula also involves longitudinal degrees of freedom and is therefore $x$ dependent, producing an $x_{f}$-dependent effect on $S_{A}$. A detailed description of our calculation will be shown in a more extended paper [27]. The nuclear $q_{T}$ smearing shown in Fig. 2(b) and in Fig. 2(d) for the $J / \psi$ (dotted curves) and in Fig. 2(d) for $\Upsilon$ (dot-dashed curve), reduces the effect of antishadowing and affects the shadowing part only marginally because of its increasing behavior with $x$ (decreasing with $x_{f}$ ). The agreement with data is substantially improved.

In Fig. 3(a) we compare the model with the E772 Drell-Yan production data [19] in the W target as a check on the role of sea quark shadowing and the effect of $Q^{2}$ evolution. The model, including a small effect from $q_{T}$ smearing (dotted curve), indeed gives a good description of the data. We show a comparison with the NA3 $J / \psi$ production data from $200 \mathrm{GeV}$ proton interactions with ${ }^{2} \mathrm{H}$ and $\mathrm{Pt}$ targets 29] in 3(b). The antishadowing region is broad with shadowing only for $x_{f}>0.4$. The width of the antishadowing peak decreases 
with increasing energy since $x_{2}$ passes from the shadowing region to the EMC regime over a smaller range of $x_{f}$. The antishadowing region is also shifted backward with $x_{f}$ as the energy grows, indicated by a comparison of $S_{A}^{\psi}$ in Fig. 2(d) and Fig. 3(b). Clearly nuclear shadowing alone, and even including the $p_{T}$-integrated spectra, does not account for the observed suppression. Other effects become important and must be taken into account [3].

Finally, we predict the shadowing of $J / \psi, \Upsilon$, and Drell-Yan production in $\mathrm{Au}+\mathrm{Au}$ collisions at RHIC energy relative to $p p$ production at the same energy in Fig. 3(c). Both the projectile and target distributions are shadowed. RHIC data will not be strongly affected by antishadowing, as expected from Fig. 1(c). Since only relatively small $x_{f}$ values can be measured, features important at large $x_{f}$ will not play a role. Thus shadowing will dominate the nuclear effects. We expect this behavior to be even more pronounced for LHC, where the range in $x$ extends below $x=10^{-3}$, Fig. $1(\mathrm{~d})$. Here recombination effects should play an important role and could be tested accurately. Calculations including recombination effects at LHC energies are in progress and will be shown elsewhere [27].

We have evaluated the effect of shadowing on heavy quarkonium production in current experiments and at RHIC. Because of their difference in mass, $J / \psi$ and $\Upsilon$ production follow different patterns. Antishadowing has been shown to be substantial at present energies, in particular, $\Upsilon$ production is within the antishadowing region. However the amount of shadowing and antishadowing for $\Upsilon$ is smaller than for the $J / \psi$ because of the perturbative $Q^{2}$ evolution of the shadowed distributions. Nuclear modifications of the primordial transverse momentum spectrum are an important addition in the low $p_{T}$ range.

Nuclear shadowing is an important feature of quarkonium production, yet at lower energies it is not sufficient. Shadowing will play a major role in nucleus-nucleus collisions, suppressing both $J / \psi$ and $\Upsilon$ production significantly but according to different patterns in $x_{f}$, over an extrapolation from $p p$ interactions.

We thank K. J. Eskola, L. Frankfurt, S. Gavin, I. Hinchcliffe, B. Kopeliovich, K.B. Luk, J. Peng, H. Schellman, M. Strikman and X.-N. Wang for useful discussions. S.L. would like to thank the Nuclear Theory Group at the Lawrence Berkeley Laboratory for their warm 
hospitality. 


\section{REFERENCES}

[1] C. Gerschel and J. Hüfner, Phys. Lett. B207 (1988) 254.

[2] S. Gavin and R. Vogt, Nucl. Phys. B345 (1990) 104. S. Gavin, H. Satz, R.L. Thews, and R. Vogt, Z. Phys. C61 (1994) 351.

[3] R. Vogt, S.J. Brodsky, and P. Hoyer, Nucl. Phys. B360 (1991) 67.

[4] S. Gavin and J. Milana, Phys. Rev. Lett. 68 (1992) 1834; S.J. Brodsky and P. Hoyer, Phys. Lett. B298 (1993) 165; D. Kharzeev and H. Satz, Z. Phys. C60, (1993) 389.

[5] A.H. Mueller and J. Qiu, Nucl.Phys. B268, 427 (1986); J. Qiu, Nucl. Phys. B291, (1988) 746 .

[6] S. Gupta and H. Satz, Z. Phys. C55 (1992) 391.

[7] M. Arneodo, CERN-PPE/92-113, to be published in Phys. Rep.

[8] T. Carroll, Thesis 1993; T. Carroll et al., FNAL Conf-93/166-E and Proceedings of the XXVII Rencontres de Moriond, March 21-27, 1993.

[9] M. Arneodo et al. (NMC Collaboration), Nucl. Phys. 371 (1992) 553.

[10] N.N. Nikolaev and B.G. Zakharov, Phys. Lett. B260 (1991) 414. S.J. Brodsky and H.J. Lu, Phys. Rev. Lett. 64 (1990) 1342.

[11] L. Frankfurt and M. Strikman, Phys. Rep. 160, 235 (1988); Nucl. Phys. B316, 340 (1989).

[12] L. Frankfurt, M. Strikman, and S. Liuti, Phys. Rev. Lett. 65 (1990) 1725.

[13] A.D. Martin, W.J. Stirling, and R.G. Roberts, Phys. Lett. B306, 145 (1993)

[14] ZEUS Collaboration, M. Derrick et al., DESY preprint 93-110, Phys. Lett. (to be published). 
[15] M.L. Mangano, P. Nason, and G. Ridolfi, Nucl. Phys. B373 (1992) 295; Nucl. Phys. B405 (1993) 507.

[16] R.K. Ellis and J.C. Sexton, Nucl. Phys. B269 (1986) 445.

[17] V.N. Gribov and L.N. Lipatov, Sov. J. Nucl. Phys. 15 (1972) 438; L.N. Lipatov, Sov. J. Nucl. Phys. 20 (1974) 181; Yu.L. Dokshister, Sov.Phys. JETP 46 (1977) 641; G. Altarelli and G. Parisi, Nucl. Phys. B216 (1977) 298.

[18] K.J. Eskola, J. Qiu and X. Wang, Phys. Rev. Lett 72, 36 (1994).

[19] D.M. Alde et al., Phys. Rev. Lett. 64 (1990) 2479.

[20] S. Liuti, L. Frankfurt, M. Strikman, and A. Yoshida, Proceedings of the XXIX Rencontres de Moriond, March 21-27, 1994, Ed. J. Tran Than Van.

[21] N.N. Nikolaev and B.G. Zakharov, Preprint KFA-IKP(Th)-1993-17, Landau-16/93, submitted to Z. für Phys. C.

[22] K.J. Eskola, Nucl. Phys. B400 (1993) 240.

[23] D.M. Alde et al., Phys. Rev. Lett. 66 (1990) 133.

[24] PHENIX Conceptual Design Report, 1993 (unpublished).

[25] ALICE Letter of Intent, CERN Report No. CERN/LHCC/93-16, 1993 (unpublished).

[26] O. Benhar et al., Phys. Lett. 177B (1986) 135.

[27] S. Liuti and R. Vogt, in preparation.

[28] D. Antreasyan et al., Phys. Rev. Lett. 48 (1982) 302.

[29] J. Badier et al., Z. Phys. C20 (1983) 101. 


\section{Figure Captions}

Figure 1. A comparison of $x_{2}$ as a function of $x_{f}$ for $J / \psi$ (solid) and $\Upsilon$ (dashed) production, at (a) $200 \mathrm{GeV}$ and (b) $800 \mathrm{GeV}$ fixed target energies and (c) RHIC and (d) LHC ion collider energies.

Figure 2. Our model calculations for $S_{A}^{\psi}$ (solid), $S_{A}^{\psi^{\prime}}$ (dashed), and $S_{A}^{\Upsilon}$ (dotted) compared to data from (a) C, (b) Ca, (c) Fe, and (d) W targets at $800 \mathrm{GeV}$ [19]. The $\Upsilon$ data is shown on (d) only. The effect of $q_{T}$ broadening in the nucleus is shown in (b) and (d) for $S_{A}^{\psi}$ (dotted) and in (d) for $\Upsilon$ (dot-dashed).

Figure 3. (a) A calculation of $S_{A}^{D Y}$ with the data from Ref. 23] at $800 \mathrm{GeV}$ on the W target (solid). The dotted curve shows the effect of $q_{T}$ broadening. (b) The $J / \psi$ production data of Ref. [29] on ${ }^{2} \mathrm{H}$ and $\mathrm{Pt}$ targets at $200 \mathrm{GeV}$ compared with $S_{A}^{\psi}$. (c) Predictions of $S_{A A}^{\psi}$ (solid), $S_{A A}^{\psi^{\prime}}$ (dashed), $S_{A A}^{\Upsilon}$ (short-dashed), and $S_{A A}^{D Y}$ (dotted) for $\mathrm{Au}+\mathrm{Au}$ collisions at RHIC. 
This figure "fig1-1.png" is available in "png" format from: http://arxiv.org/ps/hep-ph/9407272v1 
This figure "fig1-2.png" is available in "png" format from: http://arxiv.org/ps/hep-ph/9407272v1 
This figure "fig1-3.png" is available in "png" format from: http://arxiv.org/ps/hep-ph/9407272v1 